\title{
Metodología para la transformación de diseños en GRAFCET a código IEC 61131-3
}

\author{
Arantzazu Burgos, María L. Alvarez*, Nagore Iriondo, e Isabel Sarachaga \\ Departamento de Ingeniería de Sistemas y Automática, Escuela de Ingeniería de Bilbao, Universidad del País \\ Vasco/Euskal Herriko Unibertsitatea, Plaza Torres Quevedo 1, Bilbao-España. \\ (correo-e: arantzazu.burgos@ehu.eus,marialuz.alvarez@ehu.eus, nagore.iriondo@ehu.eus, \\ isabel.sarachaga@ehu.eus) \\ * Autor a quien debe ser dirigida la correspondencia
}

Recibido Jun. 5, 2020; Aceptado Ago. 5, 2020; Versión final Sep. 29, 2020, Publicado Dic. 2020

\section{Resumen}

Este trabajo presenta una metodología para la generación de código de control, adoptando estándares acordes con las actuales arquitecturas de referencia en el campo de la automatización industrial, y aplicando metodologías que siguen dichos estándares. Se genera código en lenguaje texto estructurado IEC 61131-3 partiendo de GRAFCETs de diseños obtenidos siguiendo la metodología para ingeniería de automatización (MeiA). Las fases de transformación abordan la obtención de la información necesaria de los diseños, su estructuración a un formato específico y el post-procesado para el controlador lógico programable (PLC, por sus siglas en inglés) de destino. Los resultados muestran una reducción considerable del tiempo de desarrollo y de los errores de implementación. Se concluye que la legibilidad del código obtenido en este proceso de transformación permite mejorar la comunicación entre el personal encargado del diseño y de la programación. Adicionalmente, simplifica la puesta en marcha, la modificación y el mantenimiento del código generado, y la trazabilidad entre el diseño y la implementación.

Palabras clave: automatización; sistemas de control industrial; GRAFCET; IEC 61131-3; texto estructurado

\section{Methodology for transforming GRAFCET designs into IEC 61131-3 code}

\begin{abstract}
This study presents a methodology for generating control code by applying methodologies that follow standards in accordance with current reference architectures in the industrial automation field. The code is generated in structured text language compliant to IEC 61131-3 from GRAFCET's design. This is generated following industrial automation (MeiA) methodology. The transformation phases encompass obtaining the necessary information from the designs, structuring the information into a specific format, and post-processing it for the target programmable logic controller (PLC). The results show a considerable reduction in both development time and implementation errors. It is concluded that the legibility of the code obtained in the transformation process allows improving the communication between designing and programming staff. In addition, it simplifies running, modifying, and maintaining the generated code and it facilitates tracing between design and implementation.
\end{abstract}




\section{INTRODUCCIÓN}

Actualmente, la colaboración entre aplicaciones es crucial en la ingeniería de automatización. La ingeniería y el desarrollo de los sistemas de control requieren la participación de un conjunto multidisciplinar de personas expertas que precisan entornos capaces de gestionar la colaboración en todo el ciclo de desarrollo de las aplicaciones, desde el análisis de los requisitos, pasando por el diseño hasta la implementación, puesta en marcha, operación y mantenimiento. En el nivel inferior de la jerarquía de control donde se sitúan los sistemas de control directo, en el que los controladores se conectan por un lado con la planta y por otro con niveles superiores de supervisión, tan importante es utilizar herramientas que faciliten su implementación como utilizar métodos de análisis y diseño sistemáticos (Mejia-Neira et al., 2019) que permitan desarrollar estas aplicaciones acordes con las arquitecturas de referencia RAMI 4.0 (Reference Architectural Model for Industrie 4.0), IIRA (Industrial Internet Reference Architecture), Smart Manufacturing Ecosystem o IMSA (Intelligent Manufacturing System Framework) y los estándares propuestos por ellas destacando el ANSI 95/ 88 e IEC 61131, entre otros (Fraile et al., 2019).

Este artículo se centra en el desarrollo de sistemas de control implementados sobre controladores lógicos programables (PLC - Programmable Logic Controllers) de acuerdo a la norma IEC-61131 a partir de los diseños realizados con el lenguaje de modelado GRAFCET. Dicho lenguaje de modelado es un lenguaje gráfico de especificación que permite describir el comportamiento de los sistemas de control industrial (IEC 60848, 2013). Álvarez et al. (2013) destaca su intuitiva representación gráfica, sencillez y claridad para la representación de características como el paralelismo estructural, que fundamentan su gran aceptación en el campo de la automatización, concretamente en el diseño de los mismos partiendo de las especificaciones de comportamiento. Por otro lado, el estándar IEC-61131 proporciona una plataforma abierta y común para arquitecturas de PLCs. Específicamente en su parte 3 (IEC 61131-3, 2003), propone un modelo de software, y un conjunto de lenguajes de programación tanto textuales (ST - Structured Text, IL - Instruction List) como gráficos (LD - Ladder Diagram, FBD - Function Block Diagram, SFC - Sequential Function Chart).

El trabajo presentado contribuye con una metodología y un prototipo que partiendo de los diseños del sistema de control en Grafcet permite realizar su transformación en código texto estructurado (ST) conforme a la norma IEC 61131-3. Se ha pretendido que el código generado presente una estructura clara y familiar tanto para el personal de ingeniería como para el de planta encargado de la puesta en marcha y mantenimiento de las aplicaciones, de forma que permita identificar fácilmente los diseños a partir de los que fue generado. Los diseños se han obtenido con la metodología MeiA (Methodology for Industrial Automation systems) propuesta en Álvarez et al. (2017), que ofrece guías de diseño y plantillas, incluyendo no sólo aspectos estructurales claves, sino también aspectos relacionados con la flexibilidad, modularidad y extensibilidad de los diseños que pueden ser reutilizados en otros sistemas. Siguiendo las fases y pasos de la metodología se obtienen los diseños en Grafcet que en una fase posterior se transforman en código de control en texto estructurado, lo cual reduce de forma considerable el tiempo de implementación de los programas de PLCs y produce una significativa reducción de los errores de implementación.

En los siguientes apartados se presenta, en primer lugar, un análisis de los trabajos relacionados. A continuación, la metodología que incluye el análisis y diseño del sistema de control cuyo resultado es un conjunto de Grafcets de diseño y el proceso de transformación para la generación automática del código. Posteriormente, en los resultados, se presenta un prototipo de herramienta de generación y un ejemplo de aplicación a un proceso de transferencia de piezas. En la sección de Discusión se analiza el código de control obtenido mediante el proceso de transformación y se constata la necesidad de formatos de intercambio estandarizados. Finalmente, se resumen las principales conclusiones derivadas de este trabajo.

\section{OTROS ANTECEDENTES}

La colaboración entre aplicaciones, y entre herramientas de diseño y programación que permitan la generación de software de control de manera automática, es objeto de diferentes propuestas en la bibliografía. Algunos enfoques utilizan Desarrollo Basado en Modelos (MDD - Model Driven Development) para la generación automática de código de control (Thramboulidis y Frey, 2011; Estévez y Marcos, 2011; Lukman et al., 2013; Priego, et al., 2016; Alvarez, et al., 2018). Jamro y Rzonca (2018) resalta la idoneidad de métodos y herramientas basadas en MDD para simplificar el creciente aumento del tamaño y complejidad de las aplicaciones y proponen una aproximación jerárquica y ágil para la implementación y realización de pruebas de software de control según IEC 61131-3. Las tecnologías propuestas y utilizadas para realizar el diseño en estos trabajos tienen un menor índice de implantación en la industria, ya que éstas requieren un alto nivel de conocimientos sobre las técnicas y herramientas de la ingeniería de software. A pesar de ello, se continúa haciendo esfuerzos con objeto de acercar estos métodos a la práctica industrial, como en la propuesta de González et al. (2016), que presenta un método de formalización de características de modelado de GRAFCET como base para MDD. 
Otros trabajos presentan diferentes algoritmos y métodos para la transformación del diseño a código fuente de PLC conforme a la norma IEC-61131-3. Por ejemplo, Feio (2017) propone un conjunto de técnicas de transformación de los diseños creados con una clase especial de Redes de Petri (denominada IOPT) al lenguaje LD. En el trabajo de Markiewicz et al. (2016) se presenta una herramienta que implementa un método semiautomático de transformación de FIPN (Fuzzy Interpreted Petri Net) al lenguaje ST. Díez et al. (2016) plantean una metodología de transformación de diseños GRAFCET al lenguaje IL, que estructura el código mediante instrucciones y operaciones de salto para que la estructura del programa sea más clara, sencilla y sistemática. Ahora bien, el lenguaje IL es un lenguaje de bajo nivel en comparación con los otros lenguajes del estándar, que no permite abordar la complejidad y los niveles de abstracción necesarios en los proyectos de automatización actuales, en especial en la generación de las acciones en el caso de que los sistemas de control se compongan de varios GRAFCETs.

Con el fin de conseguir coherencia entre la especificación y el programa de control, así como para obtener código de fácil comprensión y mantenimiento, Schumacher y Fay (2014) realizan un estudio de las investigaciones relacionadas con Grafcet y presentan una aproximación para la generación de código en lenguaje SFC partiendo de diseños Grafcet. Esta traducción requiere una normalización previa de los mismos para representar las estructuras jerárquicas que da como resultado Grafcets complejos y, por consiguiente, programas en SFC también complejos. Un trabajo posterior de los mismos autores propone una aproximación de transformación al lenguaje ST, manteniendo las estructuras jerárquicas (macro-etapas y enclosedgrafcets) especificadas en Grafcet sin necesidad de una normalización previa (Julius, et al., 2017). Defienden el lenguaje ST como el que mejor aborda la creciente complejidad de la programación de sistemas de control y ofrece mayor garantía de interoperabilidad entre herramientas de programación de PLCs. La selección del lenguaje está basada en criterios de equivalencia semántica, legibilidad, portabilidad y facilidad de mantenimiento, de acuerdo con las métricas propuestas por Vogel-Heuse et al. (2014). Sin embargo, el código obtenido no presenta una correspondencia directa con los Grafcets, dificultando la interpretación tanto a las personas diseñadoras como al personal de planta encargado de la puesta en marcha y mantenimiento de los proyectos de automatización. En la propuesta de Álvarez et al. (2018) se presenta una herramienta que proporciona los modelos de diseño en formato IML y genera el código en formato SFC de PLCopen XML. Pese a que SFC es el lenguaje más cercano semánticamente a Grafcet, el inconveniente reside en la dificultad de incluir la información para su representación gráfica.

La Tabla 1 resume el análisis de los trabajos comentados, que tienen como objetivo generar el código fuente del sistema de control, en base a los siguientes criterios: C1 - Estándares de diseño empleados; C2 Utilización del estándar de programación IEC 61131-3; C3 - Lenguajes de programación usados en la generación de código; y C4 - Legibilidad del código obtenido.

Tabla 1: Análisis de los trabajos relacionados

\begin{tabular}{|l|l|l|l|l|}
\cline { 2 - 5 } \multicolumn{1}{c|}{} & Diseño & IEC 61131-3 & \multicolumn{2}{l|}{ Legibilidad } \\
\cline { 2 - 6 } \multicolumn{1}{c|}{} & C1 & C3 & C4 \\
\hline Thramboulidis y Frey (2011) & SysML \& UML - IEC 61499 & $\sqrt{ }$ & - & - \\
\hline Lukman et al. (2013) & ProcGraph & $\sqrt{ }$ & FBD/ST & - \\
\hline Estévez y Marcos (2011) & CAEX \& MathML - PLCopen XML & $\sqrt{ }$ & - & - \\
\hline Priego, et al. (2016) & UML - PLCopen XML & $\sqrt{ }$ & - & - \\
\hline Feio (2017) & PN-IOPT & $\sqrt{ }$ & LD & $\checkmark$ \\
\hline Markiewicz et al. (2016) & PN-FIPN & $\sqrt{ }$ & ST & Baja \\
\hline Díez et al. (2016) & GRAFCET & $\sqrt{ }$ & IL & V \\
\hline Schumacher y Fay (2014) & GRAFCET & $\sqrt{ }$ & SFC & Baja \\
\hline Julius et al. (2017) & GRAFCET & $\sqrt{ }$ & ST & Baja \\
\hline Álvarez et al. (2018) & GRAFCET & $\sqrt{ }$ & SFC & V \\
\hline
\end{tabular}

Dicho análisis permite concluir que, si bien la reducción de tiempo y errores de implementación o la introducción de la jerarquía de los diseños se consideran importantes en todos estos trabajos, la posibilidad de modificación y mantenimiento del código generado sigue siendo un aspecto a mejorar si se pretende llevar los programas generados a la planta. Incluso la puesta en marcha puede ser compleja cuando el código no permite identificar de forma sencilla el diseño previo y hace necesario invertir tiempo y esfuerzo en la trazabilidad entre el diseño y la implementación. 


\section{METODOLOGÍA}

La metodología que se propone para el desarrollo de sistemas de control para procesos de automatización y generación de código en IEC 61131-3 tiene dos partes diferenciadas: Análisis y diseño del sistema de control y Transformación de Grafcet a texto estructurado.

\section{Análisis y diseño del sistema de control}

Para el análisis y diseño, se ha seguido la Metodología MeiA, que integra la madurez de las disciplinas de la ingeniería del software con los métodos y estándares extendidos en el ámbito de la automatización industrial. Al utilizar la terminología del dominio, se consigue una importante mejora en la comunicación entre todos los agentes involucrados en el desarrollo, puesta a punto y operación del sistema automatizado.

A lo largo del proceso de desarrollo, MeiA guía en el diseño a través de seis fases, cada una de ellas relacionada con un modo de operación: Modo automático, Modo Manual, Modo de Pruebas, Fallos, Paro de Emergencia y Producción Normal (Burgos et al., 2014). Durante el diseño incorpora el lenguaje de modelado GRAFCET para generar unidades de organización de diseño (DOUs - Design Organization Units) de tres tipos: 1) DOUs de decisión para la organización del arranque y la parada de los distintos modos de funcionamiento, así como la coordinación de todos los posibles estados del sistema. 2) DOUs de producción que realizan las operaciones de producción, coordinación de operaciones, selección de parámetros de producción, etc. 3) DOUs auxiliares que realizan procedimientos de inicialización, preparación, paros, avisos, etc. La sincronización y coordinación de los Grafcets se realiza en base a señales entre los diferentes DOUs.

\section{Transformación de Grafcet a texto estructurado}

Para obtener el código de PLC conforme a la norma IEC 61131-3 a partir de los DOUs, se deben seguir una serie de pasos de transformación como se muestra en la Fig. 1. Se requiere disponer de un editor de Grafcet con una salida a texto que proporcione toda la información implícita en los diseños desarrollados. A partir de la información textual se realiza una primera fase de pre-procesado, con el fin de obtener un formato específico para estructurar toda la información necesaria de los diseños que se deberá transformar, mediante el generador, en código ST. Por último, será necesaria una fase final de post-procesado que, dependiendo del PLC en el que se ejecutará el sistema de control y su grado de conformidad con el estándar conllevará diferentes transformaciones.

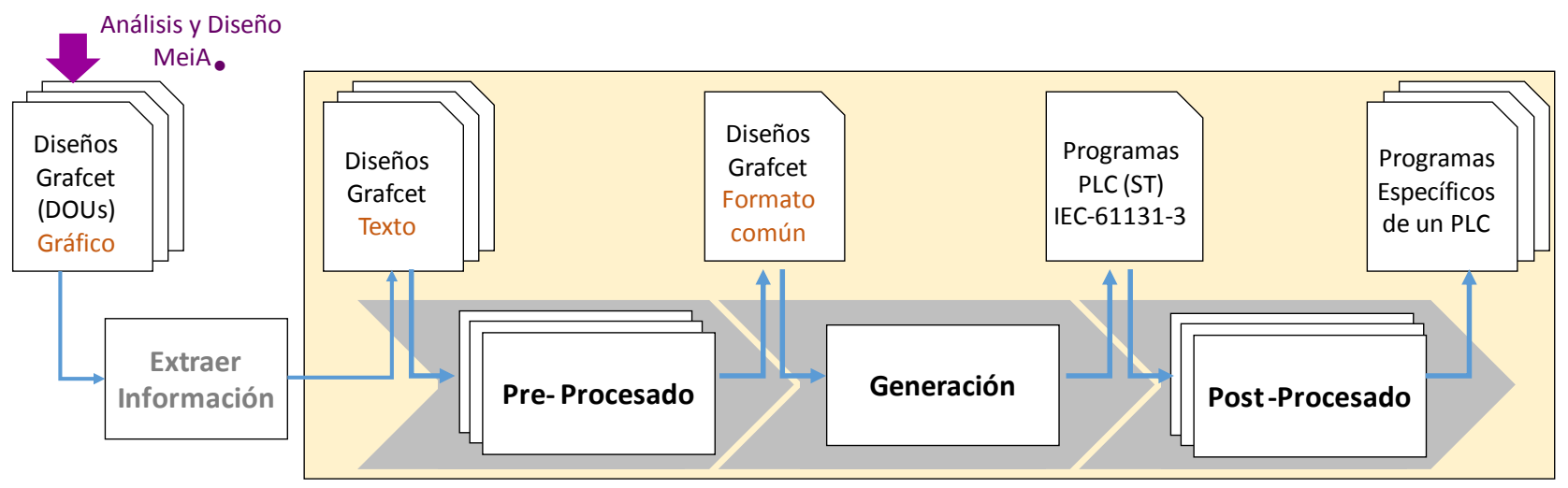

Fig. 1: Etapas de transformación de diseño a código

\section{Fase de Pre-Procesado}

La fase de pre-procesado consiste en estructurar toda la información de los diseños (Grafcets Tecnológicos) en un formato específico que en la siguiente fase se transformará en código ST. En los Grafcets desarrollados a nivel tecnológicos se distingue: la Parte Secuencial que proporciona la evolución del Grafcet, es decir, la secuencia de activación y desactivación de las distintas etapas, y la Parte Combinacional que representa las acciones a realizar en función de las etapas activas en cada momento. Además, se identifican todas las Variables definidas tanto en la parte secuencial como en la combinacional.

A la hora de realizar el pre-procesado de la Parte Secuencial se han definido dos conjuntos de reglas que debe cumplir un Grafcet. Por un lado, el conjunto de reglas estructurales: R1) Toda etapa tiene un predecesor y un sucesor. Una etapa puede tener como predecesor una transición, una divergencia en $Y$ o una convergencia en $\mathrm{O}$. Una etapa puede tener como sucesor una transición, una convergencia en $Y$ o una divergencia en O; R2) Toda transición tiene un predecesor y un sucesor. Una transición puede tener como 
predecesor una etapa, una convergencia en $\mathrm{Y}$ o una divergencia en $\mathrm{O}$. Una transición puede tener como sucesor una etapa, una divergencia en $Y$ o una convergencia en $O$; R3) Una divergencia en $Y$ tiene como predecesor una transición y como sucesor tantas etapas como ramas en la divergencia; R4) Una convergencia en $Y$ tiene como predecesor tantas etapas como ramas confluyan y como sucesor una transición; R5) Una divergencia en $\mathrm{O}$ tiene como predecesor una etapa y como sucesor tantas transiciones como ramas en la divergencia; R6) Una convergencia en $\mathrm{O}$ tiene como predecesor tantas transiciones como ramas confluyan y como sucesor una etapa. Por otro lado, un conjunto de reglas de evolución: R7) Una etapa se activa cuando está activada la etapa o etapas anteriores y se cumplen las condiciones de transición entre ambas; R8) Una etapa se desactiva cuando se cumplen las condiciones de transición a la(s) siguiente(s) etapa(s) y dicha transición se haya efectuado.

Para cada Grafcet, se procesa la información que permite crear la "Tabla de Set-Reset de las etapas" donde se presentan las condiciones que se deben cumplir para la activación y desactivación de cada una de las etapas del Grafcet de acuerdo a las reglas estructurales y de evolución mencionadas. Cada etapa tiene asociado un bit (Xi) que indicará su estado de activada/desactivada. En lo que respecta a las transiciones, cada una lleva asociada una condición, denominada receptividad (Ti_n). Además, se definen dos variables auxiliares, Init y Reset, la variable Init con el fin de poder activar las etapas iniciales y la variable Reset para desactivar todas las etapas.

En la Fig. 2 se muestra un Grafcet (Gejemplo), y en la Tabla 2 su correspondiente Tabla de Set-Reset de las etapas. Por ejemplo, la etapa 2 (X2) se activará cuando estando activa la etapa 1 (X1) se cumpla la condición asociada a la transición T1_23, y se desactivará cuando se active la etapa 5 (X5) o cuando se active la etapa 6 (X6), dependiendo de la evolución del Grafcet. Se observan, además, la condición Init para poder activar las etapas iniciales del Grafcet y la condición Reset para desactivar todas las etapas.

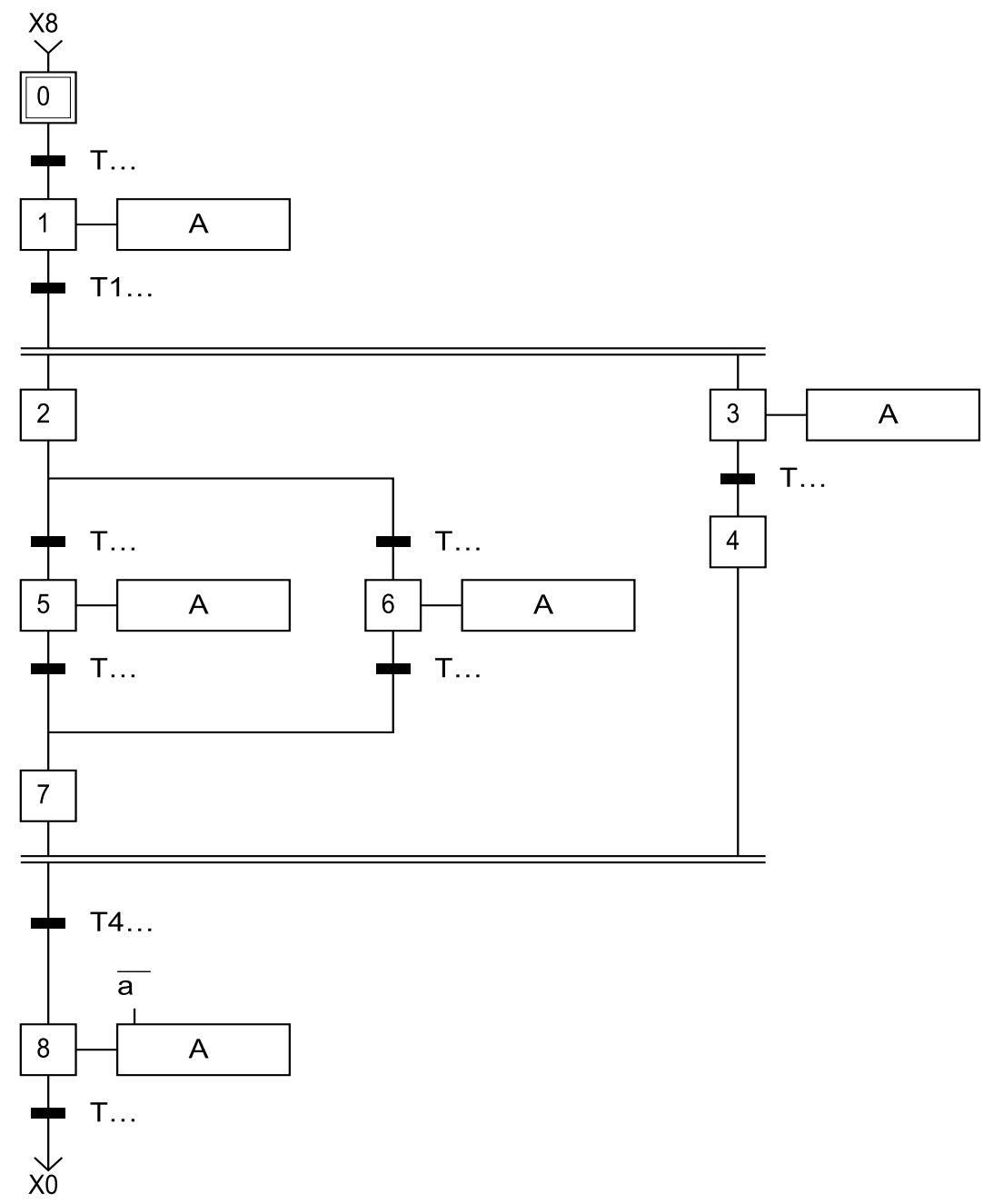

Fig. 2: Ejemplo de Grafcet (Gejemplo) 
Tabla 2: "Tabla Set-Reset de las etapas" del Grafcet Gejemplo

\begin{tabular}{|c|c|c|}
\hline Etapa & $\begin{array}{c}\text { Set } \\
\text { (Activación de Etapas) }\end{array}$ & $\begin{array}{c}\text { Reset } \\
\text { (Desactivación de Etapas) }\end{array}$ \\
\hline X0 & $\mathrm{SX0}=\mathrm{X} 8 . \mathrm{T} 8 \_0+$ Init & $\mathrm{RX} 0=\mathrm{X} 1+$ Reset \\
\hline $\mathrm{X} 1$ & $S X 1=X 0 . T 1 \_23$ & $\mathrm{RX} 1=\mathrm{X} 2 . \mathrm{X} 3+$ Init + Reset \\
\hline $\mathrm{X} 2$ & $\mathrm{SX} 2=\mathrm{X} 1 . \mathrm{T} 1 \_23$ & $\mathrm{RX} 2=\mathrm{X} 5+\mathrm{X} 6+$ Init + Reset \\
\hline X3 & $S X 3=X 1 . T 1 \_23$ & $\mathrm{RX} 3=\mathrm{X} 4+$ Init + Reset \\
\hline $\mathrm{X} 4$ & $S X 4=X 3 . T 3 \_4$ & $\mathrm{RX} 4=\mathrm{X} 8+$ Init + Reset \\
\hline X5 & $S X 5=X 2 . T 2 \_5$ & $\mathrm{RX} 5=\mathrm{X} 7+$ Init + Reset \\
\hline $\mathrm{X} 6$ & $\mathrm{SX} 6=\mathrm{X} 2 . \mathrm{T} 2 \_6$ & $\mathrm{RX} 6=\mathrm{X} 7+$ Init + Reset \\
\hline $\mathrm{X} 7$ & $\mathrm{SX7}=\mathrm{X} 5 . \mathrm{T} 5 \_7+\mathrm{X} 6 . \mathrm{T} 6 \_7$ & $\mathrm{RX} 7=\mathrm{X} 8+$ Init + Reset \\
\hline $\mathrm{x} 8$ & $\mathrm{SX} 8=\mathrm{X} 4 . \mathrm{X} 7 . \mathrm{T} 47 \_8$ & $\mathrm{RX} 8=\mathrm{X} 0+$ Init + Reset \\
\hline
\end{tabular}

Para la Parte Combinacional, se analizan las acciones identificando los distintos tipos: acciones relacionadas con actuadores del proceso (salidas), señales de control que coordinan y sincronizan los Grafcets, contadores, temporizadores, pulsos, etc. Para cada una de las acciones relacionadas con los actuadores del proceso (salidas) se realiza una búsqueda en todos los Grafcets con el fin de identificar las etapas en las que aparecen. Se considera, además, si es una acción continua, si la acción es condicional o si se trata de una asignación (set, reset o valor); las señales de control que coordinan y sincronizan los DOUs y las operaciones relacionadas con la inicialización y actualización de contadores, temporizadores, pulsos, etc., se procesan de la misma forma que las salidas.

\section{Fase de Generación}

El estándar IEC 61131-3 proporciona diversos elementos y métodos en su modelo software, que pueden agruparse en dos bloques: por un lado, los que permiten definir la arquitectura software, como son la configuración, el recurso y la tarea, y por otro los que permiten definir el software, como son las unidades de organización del programa (POU - Program Organization Units) y las variables. Un POU, puede ser de tres tipos: Función, Bloque de Función (FB - Functional Block) y Programa (PROGRAM). El POU se define por su tipo, por su interfaz o declaración de variables (locales o visibles desde el exterior) y por su cuerpo, que recoge los algoritmos programados en uno de los cinco lenguajes de programación proporcionados por el estándar.

La Parte Secuencial se implementa en POUs de tipo FB; por cada DOU obtenido en el diseño del sistema se genera un POU. En lo que respecta a la declaración de variables asociadas a los FBs, Init y Reset se definen como variables de entrada y las variables $\mathrm{Xi}$ asociadas a las etapas como variables locales o estáticas. El cuerpo de código se genera a partir de la "Tabla de Set-Reset de las etapas" que contiene las condiciones que se deben cumplir para la activación y desactivación de cada etapa. Así, para cada etapa se programan dos estructuras de tipo IF-THEN: una para evaluar las condiciones que activan la etapa y otra para evaluar su desactivación. Por otro lado, cada transición lleva una condición asociada denominada receptividad, que en los DOUs tecnológicos se enuncian como expresiones lógicas que pueden ser transformadas directamente al lenguaje ST.

La Parte Combinacional se implementa en un POU de tipo PROGRAM. En la declaración de variables se inicializan los bloques de datos asociados a los FBs desarrollados en la Parte Secuencial. En el cuerpo se instancian cada uno de esos FBs en primer lugar. A continuación se generan: 1) las acciones en función de los tipos como asignaciones para programar las señales relacionadas con los actuadores del proceso (salidas), las señales de control que coordinan y sincronizan los DOUs y las operaciones relacionadas con la inicialización y actualización de contadores, temporizadores, pulsos, etc.; 2) Los contadores, temporizadores, pulsos, etc., se implementan instanciando los FBs que proporciona la norma IEC 61131-3 y asociándoles las variables relacionadas con las operaciones de inicialización y actualización.

Por ejemplo, continuando con el Grafcet (Gejemplo) de la Fig. 2, en lo que respecta a la parte secuencial, para la etapa 2 (X2) se crearán dos estructuras de tipo IF-THEN según las condiciones de la Tabla 2 y tal y como se muestra en la Fig. 3. En cuanto a la parte combinacional asociada, la salida A2 se activará cuando esté activa la etapa 5 (X5) o cuando se active la etapa 8 (X8) si la señal a1 no está activada, tal y como queda codificado en (1). Por último, el resto de las Variables identificadas en el pre-procesado se definen como variables globales del sistema de control. 


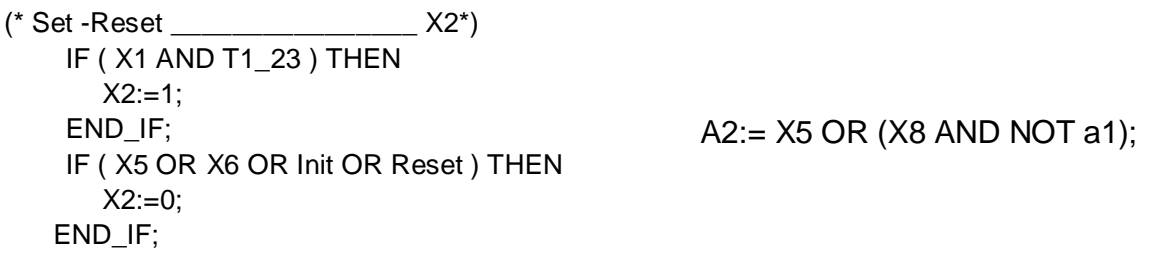

Fig. 3: Programación de la etapa X2, resaltada en la Tabla 2

\section{Fase de Post-Procesado}

El código generado en ST para el cuerpo de los POUs es el mismo para cualquier PLC, no requiere postprocesado. Sin embargo, la declaración de variables locales y globales se adapta en función del PLC destino en esta fase. En el caso de las variables locales por ejemplo, en Siemens se realiza en el interfaz asociado al POU (TIA Portal), mientras que en Beckhoff (TwinCat 2.11) se declaran etiquetadas en la zona de declaración de variables (VAR) codificada junto al cuerpo del POU. En cuanto a las variables globales, el generador las identifica, pero también será necesario especificar el tipo de variable (entrada, salida, memoria, etc.), tipo de dato (Bool, Word, etc.) y realizar la asignación de direcciones. Esta asignación de direcciones depende de la parte hardware del proyecto de automatización y deberá realizarse de forma específica. Por ejemplo, Siemens permite importar una tabla de variables en formato Excel; Pcworks (5.20.17) importa las variables de PLCOpen; en Beckhoff, las variables se definen con el operador asterisco $\left(^{*}\right)$ y posteriormente se realiza la asignación. Para cada caso, se deben proporcionan los ficheros necesarios (Grafc, XML, txt, etc.).

\section{RESULTADOS}

Para mayor claridad en la presentación, los resultados se exponen en dos subsecciones. En el primero se presenta el prototipo de la herramienta de generación de código en lenguaje ST de IEC 61131-3 y en el segundo, se describe un caso de estudio usando el prototipo en un proceso de transferencia de piezas.

\section{Prototipo}

Como entorno de desarrollo, se ha seleccionado el IDE de Eclipse. Este entorno de desarrollo de código abierto y multiplataforma proporciona un potente editor visual, un excelente motor de depuración y una interfaz gráfica de usuario flexible. Aunque Eclipse soporta múltiples lenguajes, para el desarrollo del prototipo se han utilizado Java y XML. Java proporciona código portable, mientras que XML proporciona datos portables. Como parser para documentos XML en Java se ha utilizado SAX (Simple API for XML). Este prototipo transforma los Grafcet diseñados con la metodología MeiA y realizados con el editor SFCEdit en código ST de IEC 611313. SFCEdit proporciona un sencillo interfaz gráfico y las directrices necesarias para desarrollar diagramas Grafcet conforme a la norma IEC 60848 (2013), permitiendo centrarse en el contenido, y no en el léxico y la sintaxis de Grafcet. Además, permite exportar los diseños a un formato textual en XML que será el punto de partida para la primera fase (Fase Pre-procesado).

A continuación, se presenta el esquema XML de SFCEdit que se utilizará en la generación del proyecto de automatización, así como el proyecto generado. En la Fig. 4 se muestra el esquema XML que define el léxico y sintaxis de los ficheros XML generados por SFCEdit. El elemento raíz es el proyecto (Grafcet), que se compone al menos de un Grafcet. Cada Grafcet está caracterizado por un identificador, un tipo (normal, macro o enclosure) y un comentario asociado opcional. Un Grafcet, a su vez, está formado por un conjunto de secuencias (sequences) y puede incluir enlaces horizontales (hlink) y saltos (jump) entre secuencias.

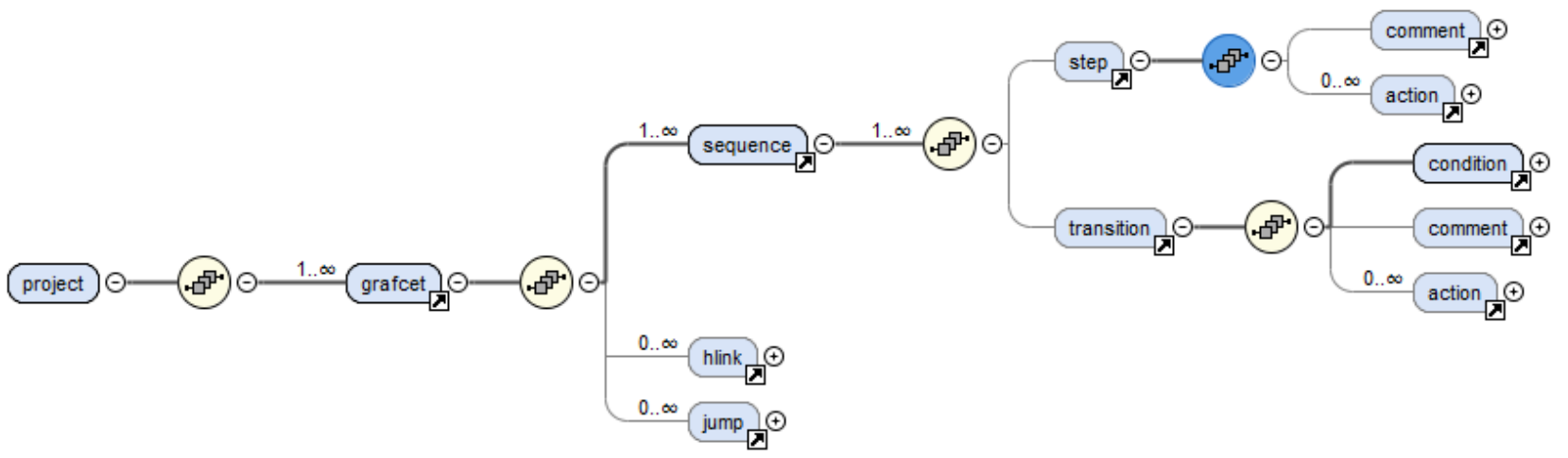

Fig. 4: Léxico y sintaxis del XML SFCEdit. 
Normalmente las secuencias están formadas por un conjunto ordenado de pasos (step) y transiciones (transition). Un paso se caracteriza por su identificador, tipo (inicial, final, macro, task, enclosing o inicial enclosing), un comentario opcional (comment) y una lista de acciones (action). Las acciones incluyen el tipo dependiendo del modo (normal, a la activación, a la desactivación, condicional, forzado, por evento) y el instante en el que se deben ejecutar, la descripción textual (text), y opcionalmente comentarios (comment) y la condición (condition) en caso de tratarse de una acción condicional. Las transiciones deben tener obligatoriamente una condición (condition), y opcionalmente un comentario (comment) y una lista de acciones (action). Los enlaces horizontales identifican la secuencia que enlazan mediante el atributo "seqid" y la lista de nodos (node) que al menos debe contener la identificación de dos secuencias "seqid". Se identifican cuatro tipos de enlaces horizontales: divergencia en $\mathrm{Y}$ (div and), divergencia en $\mathrm{O}$ (div or), convergencia en $\mathrm{Y}$ (conv and) y convergencia en $\mathrm{O}$ (conv or). Los saltos deben identificar la secuencia origen "seqid_from" y la secuencia destino "seqid_to" del salto.

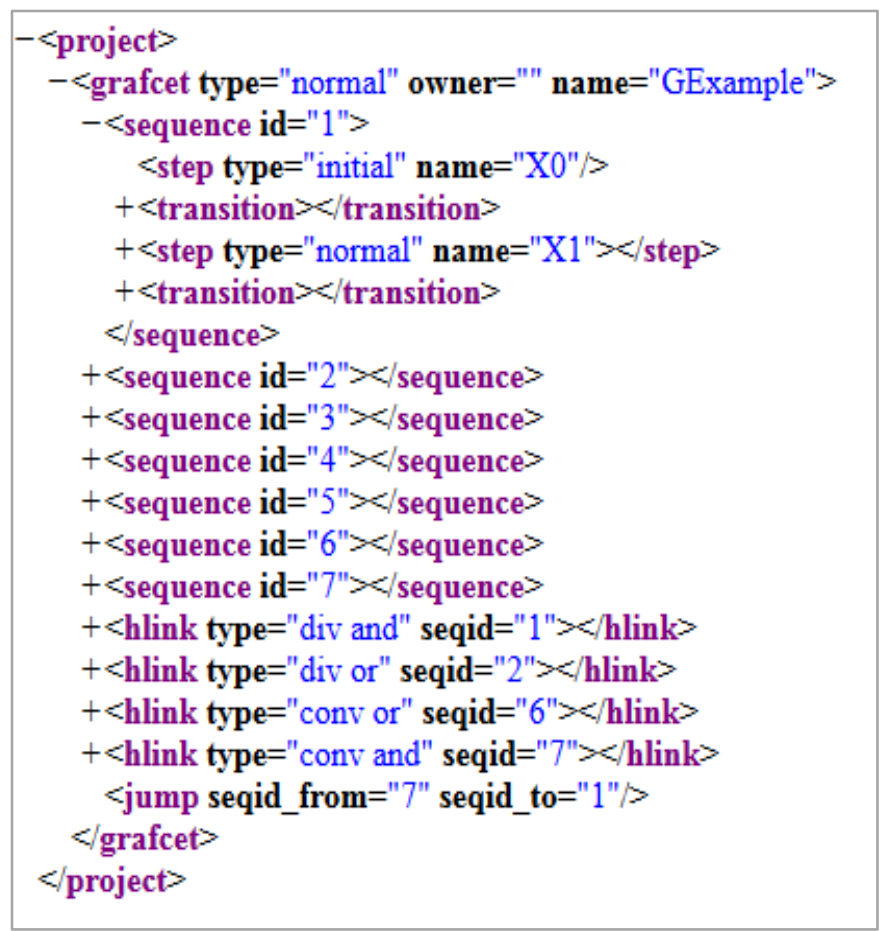

Fig. 5: XML del Grafcet para GEjemplo.

En la Fig. 5 se presenta el fichero XML generado con SFCEdit para el Grafcet GEjemplo anterior, y en la Fig. 6 se identifican gráficamente las distintas secuencias (rectángulos grises), los tipos de enlaces horizontales (div and, conv and, div or y conv or) con sus conexiones y el código XML correspondiente a una secuencia, un enlace horizontal (conv or) y un salto. La generación del código comienza con la validación del fichero XML mediante el esquema ValidarGrafcet.xsd, que presenta dos restricciones al esquema propuesto por SFCEdit: 1) siempre va a existir al menos un salto, y 2) las transiciones no disponen de lista de acciones. Partiendo del fichero validado se genera el proyecto de automatización, que consta de un conjunto de POUs de tipo FB correspondientes a la Parte Secuencial, un POU principal de tipo PROGRAM de la Parte Combinacional y la definición del conjunto de Variables del proyecto.

Como ya se ha comentado en la Fase de Pre-procesado, en primer lugar, se crea la "Tabla de Set-Reset de las etapas" correspondiente a la Parte Secuencial. La parte correspondiente al Set se realiza identificando los predecesores de cada etapa. Si la etapa es el primer elemento de una secuencia, el predecesor puede ser un enlace horizontal (divergencia en $\mathrm{Y}$ o convergencia en $\mathrm{O}$ ) o un salto, teniendo que buscar la(s) transición(es) y etapa(s) en secuencia(s) predecesora(s). Pero, si la etapa no es el primer elemento de la secuencia, la transición predecesora se encuentra en la propia secuencia y la(s) etapa(s) predecesora(s) de esta transición se pueden encontrar en la propia secuencia o en secuencia(s) predecesora(s). La parte correspondiente al Reset se realiza identificando los sucesores de cada etapa. Si la etapa es el último elemento de una secuencia, el sucesor puede ser un enlace horizontal (convergencia en $Y$ o divergencia en O) o un salto, teniendo que buscar la(s) etapa(s) en secuencia(s) sucesora(s). Pero, si la etapa no es el último elemento de la secuencia, la(s) etapa(s) sucesora(s) se encuentran en la propia secuencia o en secuencia(s) sucesora(s). Una vez recopilada toda la información, ya en la Fase de Generación, se programa un bloque funcional por cada Grafcet, que consta de la declaración de variables y el cuerpo con conjunto de sentencias IF-THEN que evalúan la activación y desactivación de cada una de las etapas. 


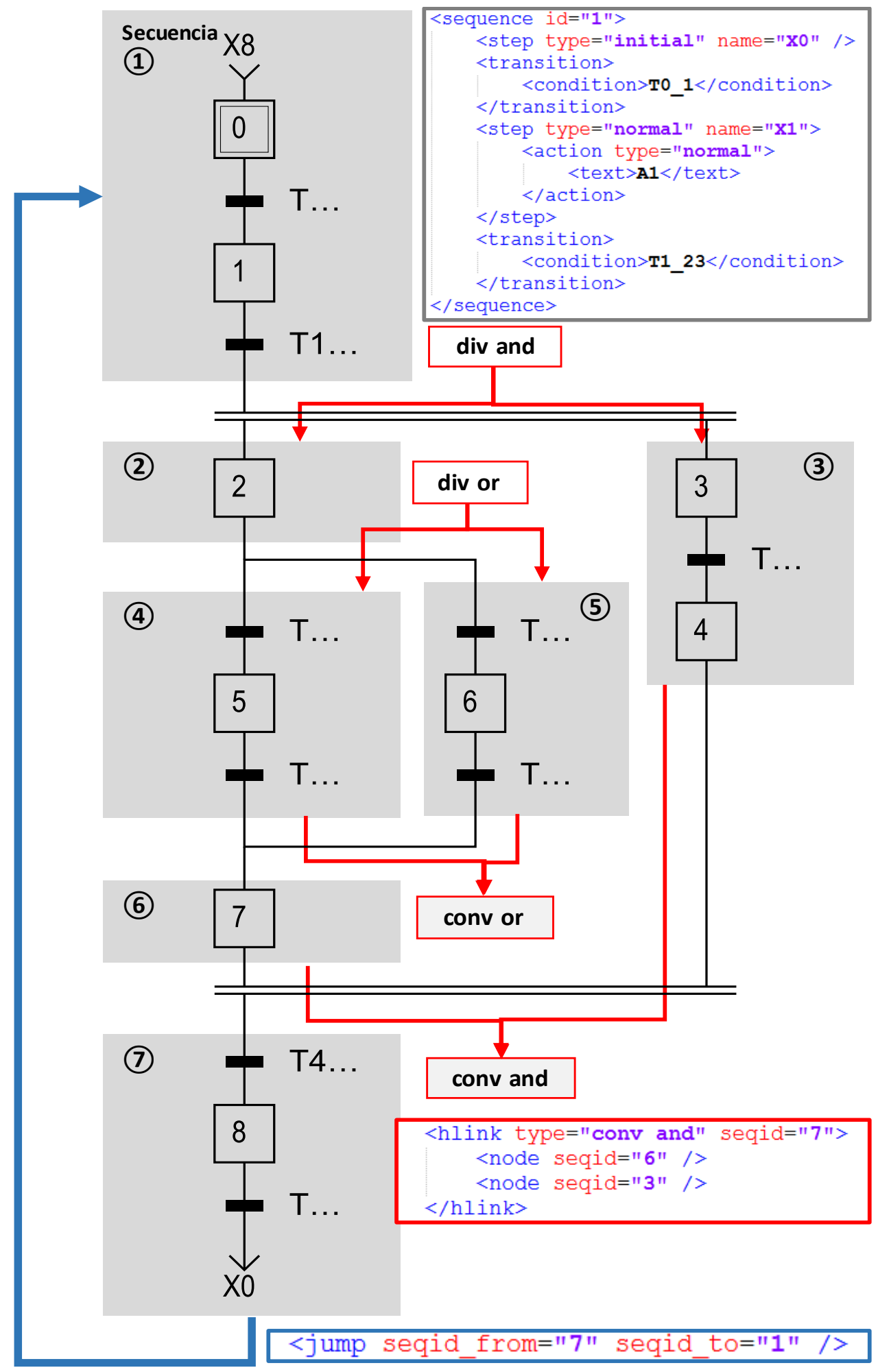

Fig. 6: Relación entre el Grafcet GEjemplo y los elementos definidos en el fichero XML.

En cuanto a la Parte Combinacional, en la Fase de Pre-Procesado de la información, para cada una de las acciones se realiza una búsqueda en el el fichero XML de todas las etapas en las que aparece dicha acción considerando, además, si es una acción continua, si la acción es condicional o si se trata de una asignación (set, reset o valor). En la Fase de Generación, para cada acción relacionada con las salidas se programa una única sentencia de asignación, en la cual figuran todas las etapas en las que aparece dicha acción (condicionada si es el caso) enlazadas por el operador OR. Para las acciones relacionadas con contadores se programa la carga de los contadores y su actualización (incremento, decremento), identificando las etapas que suscitan dichas acciones. Las acciones relacionadas con la carga y activación de los temporizadores se tratan de la misma forma. 
T Configuración del programa

Selecciona el fichero XML que deseas traducir:

C:IUserslAdmin'DesktoplTransPiezasITransferencia Piezas OCW.xml

Examinar

\section{Selecciona la carpeta donde se guardara la salida:}

C:IUsersłAdminiDesktoplTransPiezas

Examinar

Lenguaje de salida

S Structured Text (ST)

$\bigcirc$ Instruction List (IL)

Secuential Function Chart (SFC)

$\bigcirc$ Ladder Diagram (LD)

$\bigcirc$ Function Block Diagram (FBD)

Compatibilidad con

Seleccione uno:

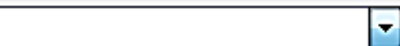

PLC TSX Micro (PL7Pro)

PLC Beckhoff (TwinCAT)

PLCOpen

\section{Iniciar}

(a)

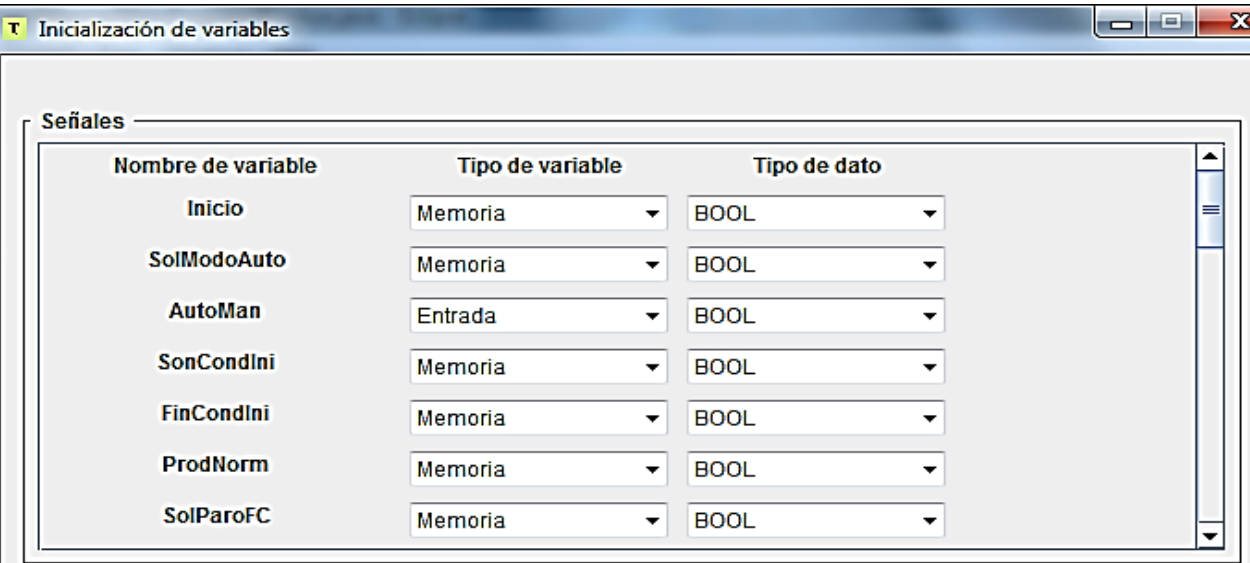

Temporizadores

\begin{tabular}{|c|c|c|}
\hline $\begin{array}{c}\text { Nombre de variable } \\
\text { Temp1 }\end{array}$ & Tipo de temporizador \\
Temp2 & TON & - \\
\hline
\end{tabular}

Contadores

Nombre de variable

ContPiezas

ContErrores

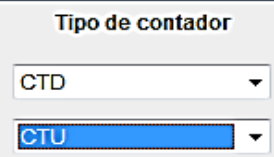

Finalizar

(b)

Volver al Menu 
En lo que respecta a las Variables del proyecto de automatización, en la Fase de Pre-Procesado, se realiza la búsqueda e identificación de las mismas en el fichero XML. Las variables de etapa son los identificadores de las mismas; las salidas, entradas y señales de control se identifican en las condiciones de las transiciones y en las acciones; $y$ las variables asociadas a los temporizadores y contadores se identifican mediante prefijos específicos establecidos en la fase de diseño (TM para temporizadores, CT para contadores). Una vez identificadas las variables, en la Fase de Post-procesado, hay que definirlas indicando el tipo de variable (entrada, salida, memoria, constante o sistema) y el tipo de dato (Bool, Word, Doubleword, etc.). Del mismo modo, es necesario indicar el tipo de contador (CTD, CTU o CTDU) y de temporizador (TON, TOF o TP). La Fig. 7a muestra el interfaz inicial que ofrece la herramienta de generación de código, que comienza con la selección del fichero XML exportado, del directorio destino de la generación y del PLC. El siguiente paso consiste en realizar la definición de variables, indicando el tipo de variable y de dato, tal y como se ha mostrado en la Fig. $7 b$.

\section{Caso de estudio}

El prototipo de la herramienta se ha aplicado en la generación del código de los ejemplos que están disponibles en el curso OCW titulado "MeiA Metodología para ingeniería de Automatización. Nivel de Diseño" (Álvarez, et al., 2018). En esta sección se presenta la correspondiente al proceso "Transferencia de Piezas", cuya vista en planta se muestra en la Fig. 8.

En este proceso, un cargador alimenta por gravedad piezas que son detectadas por un sensor (s1). El cilindro A las empuja (Amas) hasta situar la pieza frente al cilindro B (a1). Después de que el cilindro $A$ ha retrocedido (Amenos) hasta su posición inicial (a0), el cilindro B las lleva (Bmas) hasta la posición de evacuación (b1). Ambos cilindros van provistos de distribuidores de doble pilotaje. El ciclo comienza cuando se acciona un pulsador de Marcha y el sensor s1 detecta la presencia de una pieza. El proceso de transferencia se mantiene hasta que se acciona el pulsador de Paro. Si al solicitar el paro se está transportando una pieza en el sistema, se terminará de trasladar y no se moverán más piezas.

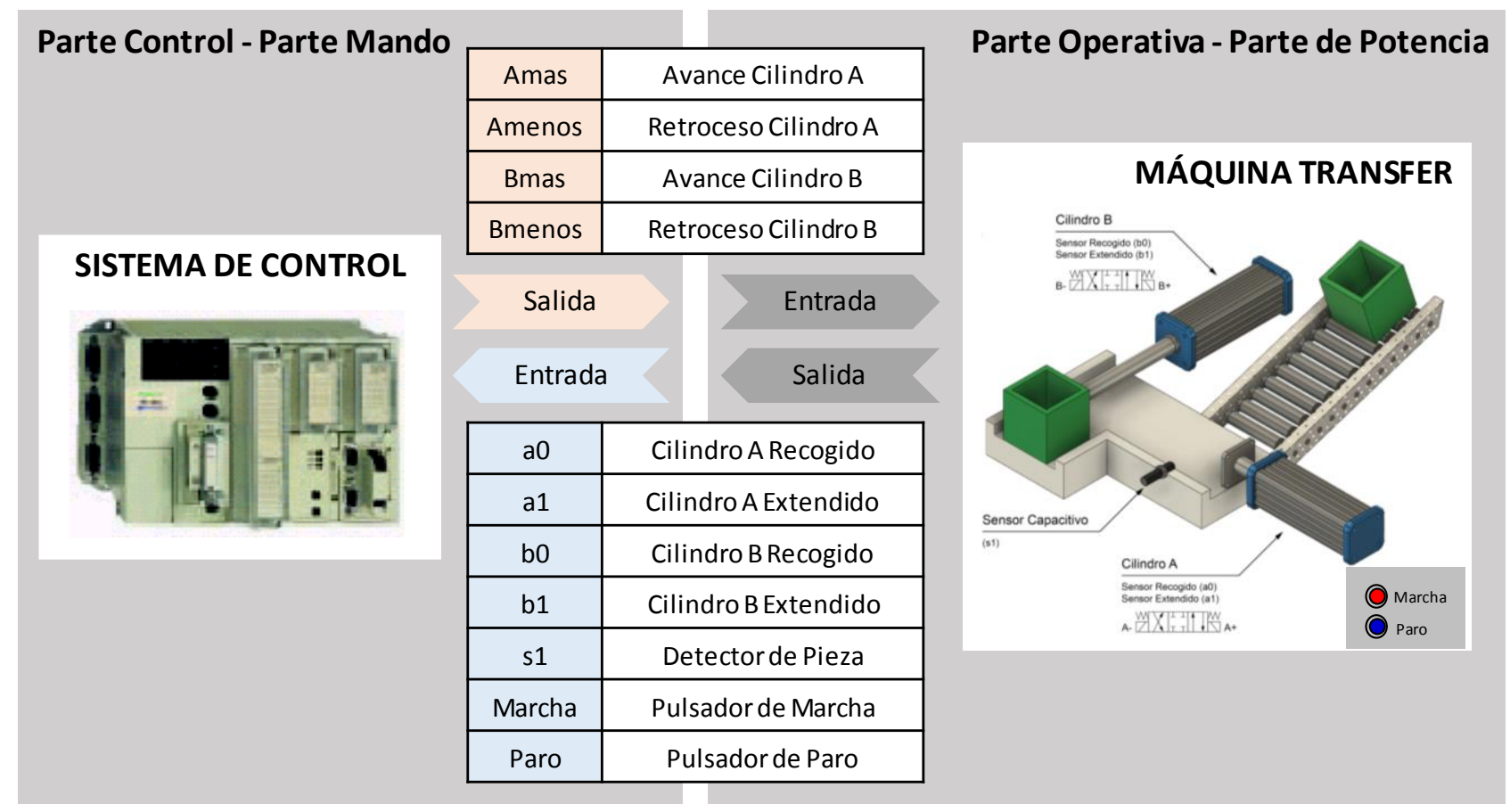

Fig. 8: Proceso de Transferencia de Piezas.

El análisis y diseño del sistema de control se ha realizado siguiendo la metodología MeiA obteniéndose cuatro DOUs (véase Fig. 9): 1) Secuencia Principal (GSecPrincipal) es un DOU de mando o decisión encargado de las formas de arranque y parada del sistema. 2) Condiciones Iniciales (GConlni) es un DOU auxiliar que se encarga de inicializar la parte operativa. 3) Producción Normal es, como su nombre indica, un DOU de producción o de base que establece la puesta en marcha de los procedimientos para la transferencia de piezas, además de cómo y en qué orden se realizan y cómo finalizan. 4) Emergencias es un DOU de mando que establece la forma de activar la parada de emergencia, las acciones (si son necesarias) previas a la parada, las acciones de señalización, los protocolos de actuación, así como las posibles acciones para la salida del modo de emergencia. 


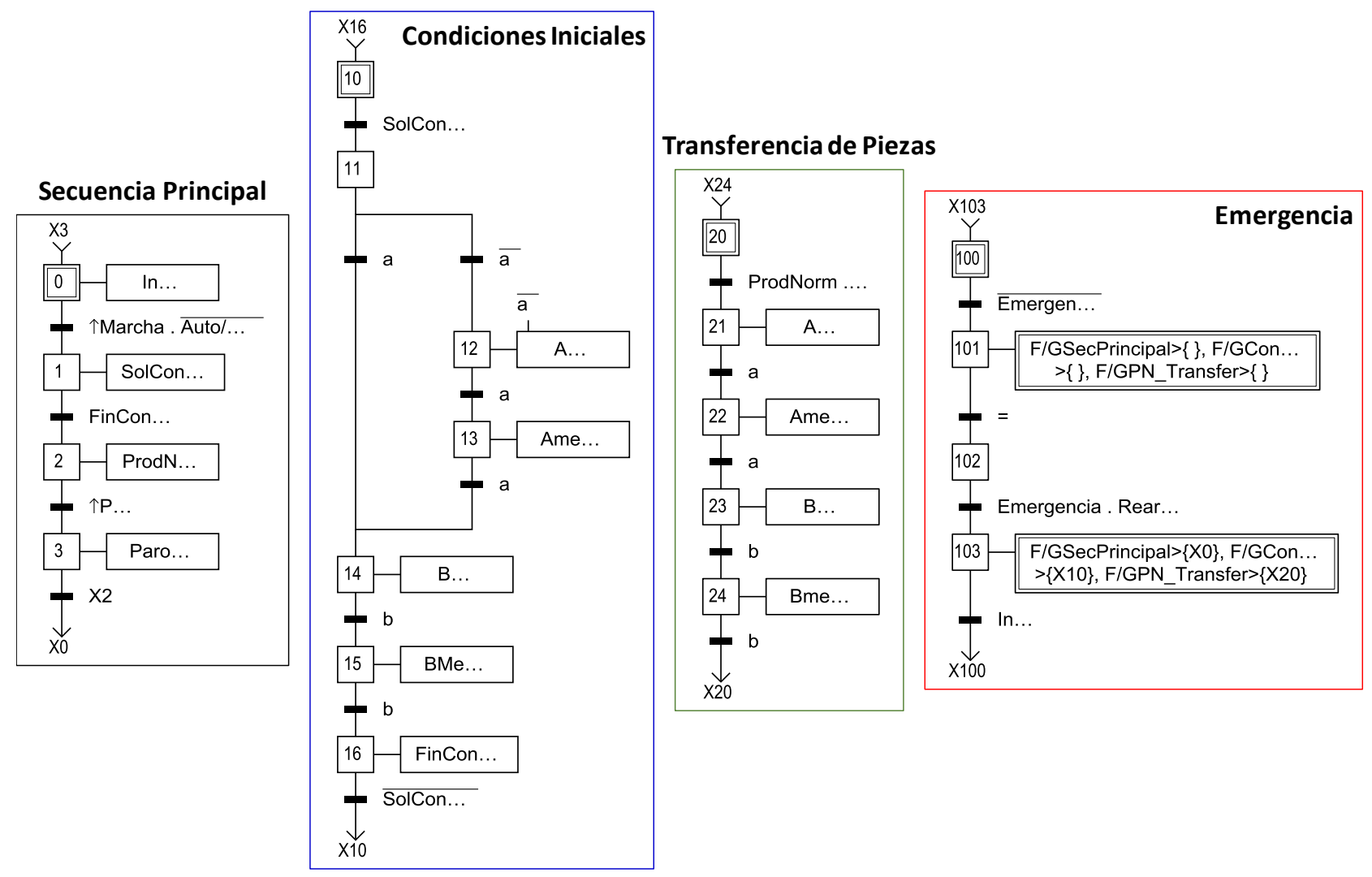

Fig. 9: Grafcets del proceso de Transferencia de Piezas.

Una vez definidas las Variables, se inicia el proceso de generación. En la Fig. 10 se presenta sólo la Secuencia Principal. En la parte superior se muestra el DOU asociado y el fichero XML exportado de SFCEdit. En la parte inferior, el código generado por el prototipo, que incluye el FB de la Parte Secuencial, así como el PRG de la Parte Combinacional y definición de Variables correspondientes a este DOU.

\section{DISCUSIÓN}

El trabajo presentado garantiza la consecución de los criterios C1-C4 (C1 - Estándares de diseño empleados C2 - Utilización del estándar de programación IEC 61131-3; C3 - Lenguajes de programación usados en la generación de código; y C4 - Legibilidad del código obtenido) que debe satisfacer el código de control obtenido mediante procesos de transformación automatizados, y que han sido empleados en el análisis comparativo realizado con los trabajos previos relacionados y resumidos en la Tabla 1. En relación con el criterio C1, este trabajo, así como gran parte de los trabajos analizados emplean el estándar GRAFCET para realizar el diseño de los sistemas de control. Dicho estándar tiene gran aceptación en el ámbito industrial, académico y de investigación.

Respecto al criterio C2, el presente trabajo al igual que el resto de los trabajos analizados, emplea el estándar de programación IEC 61131-3. Este estándar es el adoptado por todas las arquitecturas de referencia que persiguen la automatización de procesos en el marco de la fábrica del futuro. Para satisfacer el criterio C3, se ha elegido el lenguaje ST para poder abordar la complejidad de los proyectos de automatización actuales, permitiendo elevar los niveles de abstracción necesarios para acometer dichos desarrollos. Además, este lenguaje estructurado está disponible para la mayoría de los fabricantes y sus gamas de PLCs conformes al estándar IEC 61131-3.

En cuanto al criterio C4, la legibilidad del código obtenido es el aspecto más destacable del presente trabajo. Una de las principales ventajas que aporta este método de generación de código de control, es que los POUs obtenidos presentan una estructura sencilla de entender por programadores de PLCs. Esto permite que, a partir de dicho código, se puedan identificar de manera clara los diseños Grafcet de origen, facilitando, por lo tanto, las labores de puesta en marcha, modificación y mantenimiento del mismo. Respecto a la jerarquía de los diseños que otros autores solucionan mediante estructuras tipo macro y/o encapsulación, en este trabajo no se han considerado, puesto que los diseños generados con la metodología MeiA resuelven la estructuración por medio de señales de sincronización y coordinación establecidas entre los diferentes Grafcets. 
Por otra parte, en lo que respecta al proceso de transformación hay que indicar que las Fases de Preprocesado y Post-procesado son dependientes de las herramientas de diseño e implementación respectivamente. El formato textual de los diseños de Grafcet de entrada a la Fase de Pre-procesado es diferente en función del editor empleado, lo que obliga a realizar un pre-procesado diferente para cada formato, debido a que no existe un formato de intercambio estandarizado que permita la interoperabilidad real entre editores de Grafcet. De modo similar sucede en la Fase de Post-procesado siendo necesario atender a las particularidades del PLC destino.

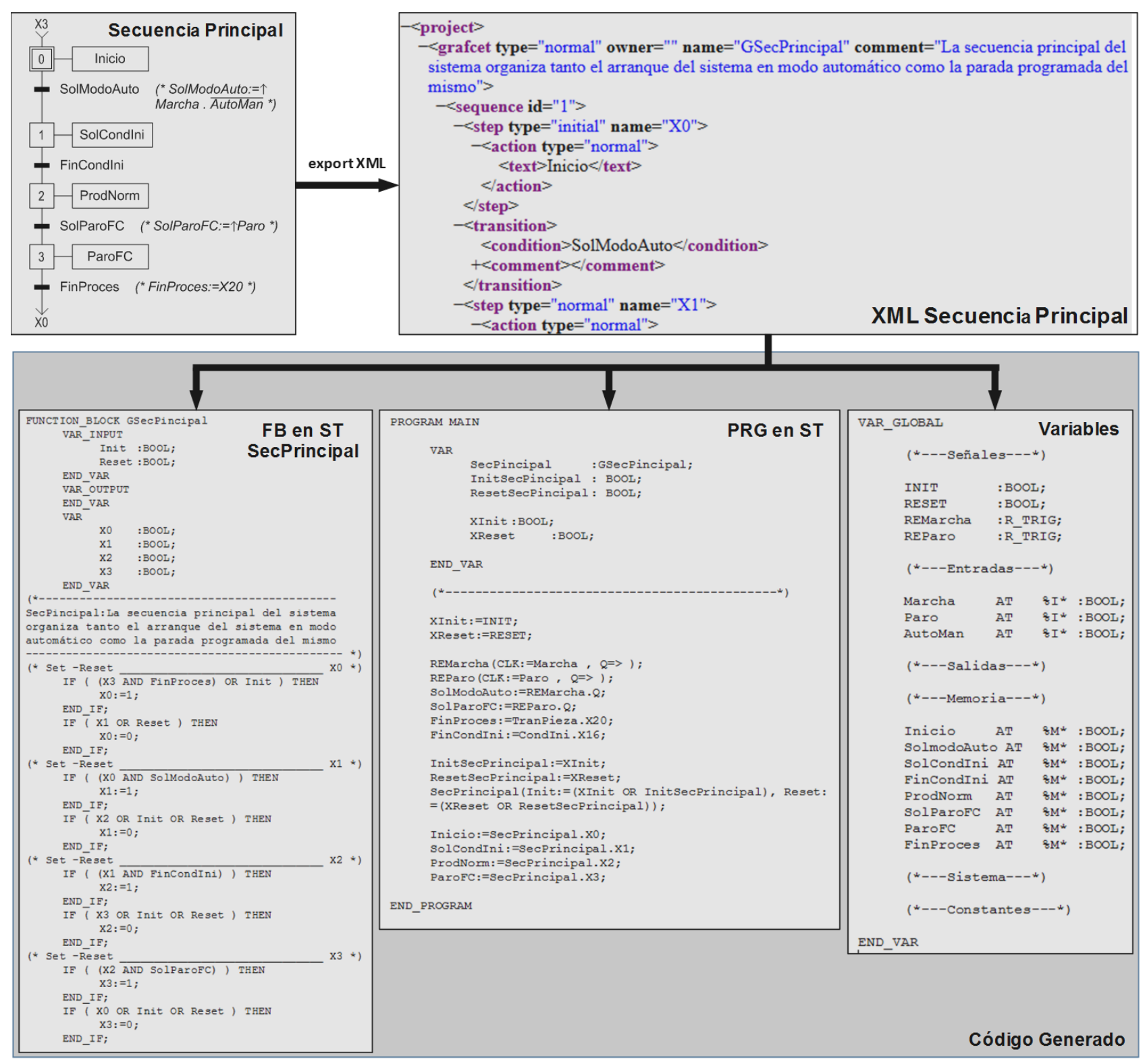

Fig. 10: Generación de código de la Secuencia Principal del proceso de Transferencia de Piezas

\section{CONCLUSIONES}

La complejidad de los sistemas de automatización actuales precisa de una metodología que guie su desarrollo a través del análisis, diseño y generación del proyecto de automatización. La automatización de esta última fase, objeto del presente trabajo, permite: 1) reducir de forma considerable el tiempo de desarrollo de los proyectos de automatización; y 2) minimizar significativamente los errores de implementación. Además, la legibilidad del código obtenido en este proceso de transformación permite: 3) mejorar la comunicación entre el personal encargado del diseño y de la programación; 4) simplificar la puesta en marcha, modificación y mantenimiento del código generado; y 5) realizar la trazabilidad entre el diseño y la implementación.

\section{AGRADECIMIENTOS}

Este trabajo está financiado por el GV/EJ, grupo A IT1324-19. 


\section{REFERENCIAS}

Álvarez, M.L., Burgos, A., Sainz de Murieta, J. y Sarachaga, M., MeiA. Metodología para Ingeniería de Automatización. Nivel de Diseño, Curso OCW - EHU/UPV, Bilbao, España (2017).

Álvarez, M.L., Estévez, E., Sarachaga, I. y otras autoras, A Novel Approach for Supporting the Development Cycle of Automation Systems, https://doi.org/10.1007/s00170-013-4793-4, International Journal of Advanced Manufacturing Technology, 68, 711-725 (2013).

Álvarez, M.L., Sarachaga, I., Burgos, A. y otras autoras, A Methodological Approach to Model-Driven Design and Development of Automation Systems, https://doi.org/10.1109/TASE.2016.2574644, IEEE Transactions on Automation Science and Engineering, 15(1), 67-79 (2018).

Burgos, A., Sarachaga, I., Álvarez, M.L., y otras autoras, Training proposal based on MeiA to face automation challenges, The International journal of engineering education, 30(5), 1254-1270, (2014).

Díez, J. M., Montoya, M. y Blasco, P. A., Metodología para la Elaboración de los Programas a Implementar en Autómatas Programables. MEPUS, Revista Iberoamericana de Automática e Informática Industrial, 13, $322-32$ (2016).

Estévez, E. y Marcos, M., Model-based Validation of Industrial Control Systems, http://doi.org/10.1109/TII.2011.2174248, IEEE Transactions on Industrial Informatics, 8(2), 302-310 (2011).

Feio, R., Translating IOPT Petri Net Models into PLC Ladder Diagrams, ICIT IEEE Int. Conference on Industrial Technology, 1211-1216, Toronto, Canada (2017).

Fraile, F., Sanchis, R., Poler, R. y Ortíz, A., Reference Models for Digital Manufacturing Platforms, https://doi.org/10.3390/app9204433, Applied Scinces, 9(20), 4433, (2019).

González, E., Marichal, R. y Hamilton, A., Ontology-Based Approach to Basic Grafcet Formalization, Journal of the Chinese Institute of Engineers, 39(8), 946-953, (2016).

IEC 60848: Int. Electrotechnical Commission, GRAFCET Specification Language for Sequential Function Charts (2013).

IEC 61131-3: Int. Electrotechnical Commission, IEC 61131-3, Programmable Controllers, Part 3: Programming Languages (2003).

Jamroa, M. y Rzoncab, D., Agile and hierarchical round-trip engineering of IEC 61131-3 control software, https://doi.org/10.1016/j.compind.2018.01.004, Computers in Industry, 96, 1-9, (2018).

Julius, R., Schürenberg, M., Schumacher, F. y Fay, A., Transformation of GRAFCET to PLC Code Including Hierarchical Structures, https://doi.org/10.1016/j.conengprac.2017.03.012, Control Engineering Practice, 64, 173-194 (2017).

Lukman, T., Godena, G., Gray J. y otros autores, Model-Driven Engineering of Process Control Software-Beyond DeviceCentric Abstractions, https://doi.org/10.1016/j.conengprac.2013.03.013, Control Engineering Practice, 21(8), 1078-1096 (2013).

Markiewicz, M., Surdej, L. y Gniewek, L., Transformation of a Fuzzy Interpreted Petri Net Diagram into Structured Text Code, $21^{\text {st }}$ MMAR- IEEE Int. Conference on Methods and Models in Automation and Robotics, 94-99, Miedzyzdroje, Polonia (2016).

Mejía-Neira, A., Jabba, D., Carrillo, G. y Calcedo-Ortíz, J., Influencia de la Ingeniería de Software en los Procesos de Automatización Industrial, http://doi.org/10.4067/S0718-07642019000500221, Información Tecnológica, 30(5), 221-230 (2019).

Murillo, L., Redes de Petri: Modelado e Implementación de Algoritmos para Autómatas Programables, Tecnología en Marcha, 21(4), 102-125 (2008).

PLCopen, Organization for Efficiency in Automation, Holanda, https://www.plcopen.org/ (1992).

Priego, R., Armentia, A., Estévez, E., Marcos, M., Modeling techniques as applied to generating tool-independent automation projects, https://doi.org/10.1515/auto-2015-0072, at - Automatisierungstechnik, 64(4), 325-340 (2016).

Provost, J., Roussel, J. y Faure, J., Translating Grafcet Specifications into Mealy Machines for Conformance Test Purposes, https://doi.org/10.1016/j.conengprac.2010.10.001, Control Engineering Practice, 19(9), 947-957 (2011).

Schumacher, F. y Fay, A., Formal Representation of GRAFCET to Automatically Generate Control Code, https://doi.org/10.1016/j.conengprac.2014.09.008, Control Engineering Practice, 33, 84-93 (2014).

Thramboulidis, K. y Frey, G., Towards a Model-Driven IEC 61131-Based Development Process in Industrial Automation, https://doi.org/10.4236/jsea.2011.44024, Journal of Software Engineering and Applications, 4(4), 217-226 (2011).

Viatkyn, V., Software Engineering in Industrial Automation: State-of-the-Art Review, https://doi.org/10.1109/TII.2013.2258165, Transactions on Industrial Informatics, 9(3), 1234-1249 (2013).

Vogel-Heuse, B., Diedrich, C., Fay, A. y otros autores, Challenges for Software Engineering in Automation, https://doi.org/10.4236/jsea.2014.75041, Journal of Software Engineering and Applications, 7(5), 440-451 (2014). 